\title{
Turbulence-Flame Interactions in Lean Premixed Dodecane Flames
}

\author{
A. J. Aspden ${ }^{1,2}$, J. B. Bell ${ }^{2}$, M. S. Day ${ }^{2}$ and F. N. Egolfopoulos ${ }^{3}$ \\ ${ }^{1}$ Mathematical Sciences, University of Southampton, Southampton, Hampshire, SO17 1BJ, UK \\ ${ }^{2}$ Center for Computational Sciences and Engineering, Lawrence Berkeley National Laboratory, Berkeley, CA 94720, USA \\ ${ }^{3}$ University of Southern California, Department of Aerospace and Mechanical Engineering, Los Angeles, CA 90089, USA
}

\begin{tabular}{|c|c|c|}
\hline \multirow{6}{*}{$\begin{array}{r}\text { corresponding author: } \\
\text { address: }\end{array}$} & \multicolumn{2}{|l|}{ M. S. Day } \\
\hline & \multicolumn{2}{|c|}{ Center for Computational Science } \\
\hline & \multicolumn{2}{|c|}{ Lawrence Berkeley National Labc } \\
\hline & \multicolumn{2}{|c|}{ Mailstop 50A-1148 } \\
\hline & \multicolumn{2}{|c|}{ One Cyclotron Road } \\
\hline & \multicolumn{2}{|c|}{ Berkeley, CA 94470} \\
\hline email: & \multicolumn{2}{|c|}{ MSDay@lbl.gov } \\
\hline colloquium: & \multicolumn{2}{|c|}{ Turbulent flames } \\
\hline keywords: & \multicolumn{2}{|c|}{$\begin{array}{l}\text { Turbulent premixed flames, direc } \\
\text { simulation, detailed chemistry, } \\
\text { flow, dodecane }\end{array}$} \\
\hline & \multicolumn{2}{|c|}{ Word count using method 2} \\
\hline & Table 1 & 55 \\
\hline & Figure 1 & 132 \\
\hline & Figure 2 & 409 \\
\hline & Figure 3 & 143 \\
\hline & Figure 4 & 169 \\
\hline & Figure 5 & 154 \\
\hline & Figure 6 & 187 \\
\hline & Figure 7 & 330 \\
\hline & Figure 8 & 240 \\
\hline & Figure 9 & 178 \\
\hline & Figure 10 & 233 \\
\hline & - & - \\
\hline \multicolumn{2}{|c|}{ Tables and figures } & 2230 \\
\hline \multicolumn{2}{|c|}{ Combined text } & 3674 \\
\hline & - & 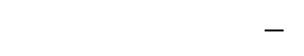 \\
\hline & Total & $5904(\max .6200)$ \\
\hline
\end{tabular}

(C) 2016. This manuscript version is made available under the Elsevier user license http://www.elsevier.com/open-access/userlicense/1.0/ 


\begin{abstract}
Turbulent lean premixed dodecane/air flames are simulated in a doubly-periodic domain using detailed kinetics and transport over a range of Karlovitz number. We observe extensive thickening of thermal profiles through the flames that increases with turbulent intensity. The high Lewis number of the flames acts to suppress wrinkling of the flame resulting in considerably lower turbulent flame speeds than is observed for lower molecular weight fuels. The impact of high Lewis number is also reflected in a negative correlation of local consumption-based flame speed with curvature. Characteristic of heavy hydrocarbons, pyrolysis of the fuel into smaller fuel fragments is separated in temperature from the primary consumpution of oxygen, which peaks at a higher temperature where the fuel fragments are consumed. The resulting intermediate species are partially entrained within the cool region ahead of the flame, but the overall pyrolysis-oxidation sequence appears essentially unaffected by turbulent mixing; however, the peak rates of these reactions are dramatically reduced.
\end{abstract}




\section{Introduction}

Detailed chemical models for the combustion kinetics of real fuels (e.g., gasoline, diesel, and jet fuels) are important tools for improving the design of combustion technologies. A key issue in such systems is the effect of turbulent mixing on combustion chemistry, which can be explored using detailed numerical simulation. However, real fuels contain variable blends of thousands of hydrocarbon compounds so the use of detailed models is prohibitive. Instead the focus is on dodecane with a molecular weight representative of heavy practical fuels. In particular, lean premixed dodecane/air combustion in a turbulent environment is investigated using numerical simulations, based on a reduced kinetic model. There is a growing body of work of direct numerical simulation of turbulent premixed flames with detailed chemistry and transport, mostly focussing on smaller fuel molecules, such as methane and hydrogen and syngas mixtures. The goal in this work is to highlight characteristics of the chemistry-turbulence interactions specific to the pyrolysis of large-molecule fuels, and compare the oxidation and heat release of the resulting fragments to similar scenarios involving the smaller, simpler fuels.

Aspden et al. [1-3] have considered hydrogen, methane and propane flames with detailed kinetics in moderate-to-intense turbulence. Carlsson et al. [4, 5] and Tanahashi et al. [6-8] have also considered turbulent premixed hydrogen and methane flames with detailed chemistry. Little work has been done on direct numerical simulation of high molecular weight hydrocarbon fuels, particularly for high Karlovitz number premixed flames. In a sequence of papers, Savard et al. [9, 10] and Lapointe et al. [11] discuss simulations of premixed heptane flames at high Karlovitz numbers with detailed chemistry and transport, building on previous work [12] based on the hydrogen simulations in [1]. The focus of these papers is on the impact of differential diffusion on flame response, even at high Karlovitz numbers; the authors note a broadening of the flame and a transition to composition versus temperature profiles characteristic of a unity Lewis number flame and a reduction in heat release and fuel consumption rates.

This paper presents simulations of lean premixed dodecane flames with detailed kinetics and transport 
at high Karlovitz number, targetting matching conditions to our previous work [3, 13], and similar in spirit to the heptane simulations discussed above, but at moderate turbulence levels. Turbulent conditions are characterised in terms of the one-dimensional unstrained steady flame properties using the Karlovitz and Damköhler numbers, which are defined by

$$
\mathrm{Ka}^{2}=\frac{\check{u}^{3}}{s_{F}^{3}} \frac{l_{F}}{l} \quad \text { and } \quad \mathrm{Da}=\frac{s_{F}}{\check{u}} \frac{l}{l_{F}}
$$

where $\breve{u}$ and $l$ are the turbulent rms velocity fluctuation and integral length scale, respectively, and $s_{F}$ and $l_{F}$ are the steady unstrained flame speed and width, respectively. In the present paper, cases are constructed with $\mathrm{Ka}$ in the range, $1-36$, following the approach detailed in $[1,14]$. An overview of the basic flame characteristics is first presented, followed by a discussion of the differences between the behaviour of dodecane and methane in comparable premixed turbulent flames.

\section{Computational Methodology}

The simulations presented here are based on a low Mach number formulation of the reacting flow equations. The methodology treats the fluid as a mixture of perfect gases; the corresponding conservation equations for an open domain are

$$
\begin{aligned}
\frac{\partial(\rho \boldsymbol{u})}{\partial t}+\nabla \cdot(\rho \boldsymbol{u u}+\tau) & =-\nabla \pi+\rho \boldsymbol{F} \\
\frac{\partial\left(\rho Y_{i}\right)}{\partial t}+\nabla \cdot\left(\rho Y_{i} \boldsymbol{u}+\mathcal{F}_{i}\right) & =\rho \dot{\omega}_{i} \\
\frac{\partial(\rho h)}{\partial t}+\nabla \cdot(\rho h \boldsymbol{u}+\mathcal{Q}) & =0
\end{aligned}
$$

where $\rho$ is the density, $\boldsymbol{u}$ is the velocity, $h$ is the mass-weighted enthalpy, $T$ is temperature. $Y_{i}$ is the mass fraction and $\dot{\omega}_{i}$ is the molar production rate for species $i$. A mixture-averaged model is assumed for diffusive transport, ignoring Dufour and Soret effects; $\tau$ is the stress tensor; $\mathcal{Q}=h_{i} \mathcal{F}_{i}-\lambda \nabla T$ is the heat flux and $\mathcal{F}_{i}=-\rho \mathcal{D}_{i} \nabla X_{i}$ is the species diffusion. The momentum source term, $\boldsymbol{F}$, is a long-wavelength forcing 
term designed to establish and maintain turbulence with the desired properties. The chemical kinetics and transport are modelled using the dodecane mechanism of You et al. [15], consisting of 56 species with 289 fundamental reactions. These evolution equations are supplemented by CHEMKIN-compatible databases for thermodynamic quantities. Transport properties are computed using EGLIB [16].

The basic discretisation combines a spectral deferred corrections (SDC) coupling of chemistry and transport [17] with a density-weighted approximate projection method for low Mach number flow [18]. The projection method implements a constrained evolution on the velocity field via the SDC iterations, which ensures that the update simultaneously satisfies the equation of state and discrete conservation of mass and total enthalpy. A time-explicit approach is used for advection; faster diffusion and chemistry processes are treated time-implicitly, and iteratively coupled together within the defferred corrections strategy. Since the low Mach system does not support acoustic waves, the time step size is governed by a CFL constraint based on advective transport. The integration algorithm is second-order accurate in space and time.

The performance of the numerical scheme for direct numerical simulation of premixed flame systems in regimes comparable to the present study was examined in [14]. An effective Kolmogorov length scale, $\eta_{\text {eff, }}$ was formulated, which measures the actual Kolmogorov length scale realised in a simulation at a given resolution. Here, the most computationally demanding simulation, having the highest turbulence levels, has a computational cell width that is approximately 1.27 times the Kolmogorov length scale, $\eta$. In this case, the numerical scheme produces $\eta_{\text {eff }} / \eta<1.03$. All other cases were better resolved.

Downward-propagating flames were simulated in a high aspect ratio domain, with periodic lateral boundary conditions, a free-slip base and outflow at the top. The momentum source term that maintains the background turbulence results in a time-dependent zero-mean velocity field. Four flames were considered at atmosphere pressure with an unburned mixture temperature of $298 \mathrm{~K}$ and an equivalence ratio of $\varphi=0.7$. For this mixture, the freely propagating flame speed $s_{F} \approx 23.6 \mathrm{~cm} / \mathrm{s}$ and thermal thickness $l_{F} \approx 520 \mu \mathrm{m}$. We note that $298 \mathrm{~K}$ is below the boiling point of dodecane at this pressure; however, the simulations are 
performed with an ideal gas equation of state that does not model condensation. The rationale for using this temperature is to facilitate comparisons with previous work on smaller fuels discussed above. As shown in [14], the turbulence forcing term gives rise to an integral length scale that is approximately one tenth of the domain size. The domain width was chosen to be $L=5.2 \mathrm{~cm}$ so that $\Lambda=l / l_{F}=1$ and $L=10 l=10 l_{F}$. An inert calculation was run to establish the turbulence, and the reacting flow simulation was initialised by superimposing a steady unstrained flame profile onto the turbulent velocity field. Four Karlovitz numbers were chosen to match previously-reported simulations of hydrogen [13] and methane [3], which were run at $\Lambda=4$ rather than $\Lambda=1$; specifically, the four cases are $\mathrm{Ka}=1,4,12$ and 36 . Note that matching simulations in hydrogen and methane at $\Lambda=1$ have also been run for comparison. The computational grid in each of the three lower Ka cases was $128 \times 128 \times 1024$, and for the $\mathrm{Ka}=36$ case was $192 \times 192 \times 1536$ to ensure that the smallest turbulence scales were sufficiently well resolved. These resolutions correspond to approximately 13 and 19 computational cells across the thermal thickness, respectively. The four cases are summarised in Table 1, and the position of these flames on the premixed regime diagram are shown in Fig. 1. Note that each simulation at the same $\mathrm{Ka}$ also has the same $\mathrm{Da}$ and therefore the same $\mathrm{Re}=\mathrm{Ka}^{2} \mathrm{Da}^{2}$, and that this definition of $\mathrm{Ka}$ is the appropriate measure of turbulent-flame interactions in the inertial subrange of the turbulent cascade [19].

\section{Results}

The basic structure of the flame is illustrated with two-dimensional slices through the three-dimensional data at $\mathrm{Ka}=36$ as shown in Fig. 2. Note the extensive thickening of the density field in the preheat zone, which can also be seen in the fuel mass fraction and temperature fields, although to a lesser extent. The fuel consumption rate and heat release present much narrower profiles. As expected for a high Lewis number fuels there is a strong negative correlation between consumption rate and flame surface curvature; high reaction rates are observed where there is strong negative curvature (where the centre of curvature is in 
the reactants). The images of fuel consumption and heat release show a distinct separation (note how the peak heat release and peak fuel consumption occur on the high-temperature and low-temperature side of the isotherm, respectively) between where the fuel is consumed and where heat is released, which illustrates an important feature of dodecane combustion.

The separation of fuel consumption and heat release is indicative of a fundamental difference between combustion of dodecane and small hydrocarbons such as methane. The primary decomposition of dodecane occurs at lower temperatures through a combination of pyrolysis and oxidation reactions. Within the context of the skeletal mechanism used in this study, dodecane decomposes into a number of relatively stable intermediates, namely, $\mathrm{CH}_{4}, \mathrm{C}_{2} \mathrm{H}_{4}, \mathrm{C}_{3} \mathrm{H}_{6}, \mathrm{C}_{4} \mathrm{H}_{8}, \mathrm{C}_{5} \mathrm{H}_{10}$ and $\mathrm{C}_{6} \mathrm{H}_{12}$. The first stage of dodecane decomposition also produces $p \mathrm{C}_{4} \mathrm{H}_{9}$ and $n \mathrm{C}_{3} \mathrm{H}_{7}$ but both are highly unstable and rapidly react to form combinations of the above molecules and $\mathrm{CH}_{2} \mathrm{O}$, which is also stable. Images of each of these species are presented in Fig. 2. Because they are stable, they can be entrained into the cool region in front of the flame as seen in the figure.

Consumption-based global turbulent flame speeds normalised by the steady unstrained flame are presented as a function of Karlovitz number in Fig. 3, where a comparison is made with simulations of hydrogen and methane flames at the same conditions. This consumption-based global turbulent flame speed is defined based on the integrated fuel consumption rate in the domain, normalised by the cross-section area,

$$
s_{T}=\frac{-1}{\left(\rho Y_{\mathrm{F}}\right)_{\mathrm{u}} L^{2}} \int_{V} \rho \dot{\omega}_{F} \mathrm{~d} V
$$

where the suffices $\mathrm{F}$ and $\mathrm{u}$ denote fuel and the unburned conditions, respectively. Note that since the simulations are all conducted at the same length scale ratio $\Lambda=l / l_{F}$, this is equivalent to plotting as a function of velocity ratio $\Upsilon=\check{u} / s_{F}$, but has been presented in terms of Ka because it is a more appropriate measure of turbulence-flame interactions (see [19] for further discussion). The vertical lines indicate one standard deviation observed during this period. There are two clear trends; turbulent flame speed increases with increasing Karlovitz number and decreasing global Lewis number. For the thermodiffusively-unstable low-Lewis-number hydrogen flames, turbulence wrinkles the flame producing regions of both positive and 
negative curvature; positive curvature leads to a local increase in flame speed and the formation of a dominant leading edge, which is compounded by an increase in flame surface area, giving turbulent flame speeds an order of magnitude faster than the steady unstrained flame [1, 13]. For the thermodiffusively-neutral unity-Lewis-number methane flames, there is only a slight variation of local flame speed with curvature, so the enhanced flame speed results predominantly from an increase in flame surface area due to turbulence [3]. For the high-Lewis-number dodecane flames, the response to turbulence is quite different; turbulence again produces both positively and negatively curved regions. The local flame speed is enhanced in regions of negative curvature, but cannot form a leading edge in the same way as the hydrogen flames. The positively curved regions experience a defocussing of heat through diffusion, which results in a decrease in reaction rates. This decrease increases the chemical time scale, decreasing the local Damköhler number and increasing the local Karlovitz number, which makes the flame locally more susceptible to turbulent mixing; turbulence can mix partially reacted fluid into the preheat zone, which results in a pronounced thickening. The overall result on the flame speed is that it is marginally larger than that of the steady unstrained flame at low Ka and only increases by about $50 \%$ at $\mathrm{Ka}=36$.

To obtain a more quantitative measure of the curvature dependence of the flame, joint probability density functions (JPDFs) were computed of consumption-based local flame speed and flame surface mean curvature (calculated as the mean of principal curvatures; see [20] for details), which are presented in Fig. 4. To evaluate the local flame speed, the flame surface was identified with the $1460 \mathrm{~K}$ isotherm (the temperature corresponding to peak fuel consumption in the steady unstrained flame), and the fuel consumption rate was integrated in a local coordinate system constructed following temperature gradients away from the surface, following the procedure detailed in [20],

$$
s_{T}^{c}=\frac{-1}{\left(\rho Y_{\mathrm{F}}\right)_{\mathrm{u}} A_{\mathrm{ref}}} \int_{\Omega} \rho \dot{\omega}_{\mathrm{F}} \mathrm{d} \Omega
$$

where $\Omega$ is prism volume, and $A_{\text {ref }}$ is the area of intersection between the $\Omega$ and the flame surface (note this differs from equation (2) in the volume of integration and reference area). The JPDF for Ka $=1$ shows the 
negative correlation expected from a high Lewis number flame. The JPDF for Ka $=36$ shows a much wider range of responses and the negative correlation is less pronounced. In addition, the average consumptionbased flame speed at zero curvature is considerably less than that of the steady unstrained flame; reflecting the increased chemical time scale as a result of turbulent mixing.

The impact of turbulent mixing on the flame is reflected in the thickening of the preheat zone that was illustrated in Fig. 2. To quantify the broadening of the preheat zone, a thickening factor is defined (following [3]) by taking the gradient of the temperature field, evaluating the conditional mean as a function of temperature (the conditional variable is denoted by $\xi$ ), and normalising by the corresponding profile from the $\mathrm{Ka}=1$ flame,

$$
\Theta_{n}(T)=\frac{\left\langle\left.\nabla T\right|_{T}\right\rangle_{\mathrm{Ka}=1}}{\left\langle\left.\nabla T\right|_{T}\right\rangle_{\mathrm{Ka}=n}}
$$

Note the normalisation by the $\mathrm{Ka}=1$ flame is used because the steady unstrained flame for hydrogen is not particularly relevant [21]. This choice has little effect for methane and dodecane. The thickening factors for the dodecane flames, along with the comparable results for methane and hydrogen flames, are presented in Fig. 5. For both methane and dodecane the degree of thickening increases with increasing Karlovitz number; however, the thickening is more pronounced for dodecane - over $50 \%$ broader across most of the flame at $\mathrm{Ka}=36$. In contrast, the hydrogen flames actually get thinner, increasingly so over this range of Ka. A particularly interesting feature is the bump in the dodecane profiles, which corresponds to the temperature at peak heat release (and hence maximal gradient of temperature). We speculate that this is a signature of turbulence penetrating the reaction zone, and so the broadening of the flame at this temperature is more prounounced because of the magnitude of the temperature gradient in the reference flame.

One common feature of smaller-hydrocarbon flames exploited in experiments (e.g. [22]) is the correlation between heat release and the product of the mole fractions of $\mathrm{OH}$ and $\mathrm{CH}_{2} \mathrm{O}$. Fig. 6 presents the JPDF of heat release and $\mathrm{X}_{\mathrm{OH}} \times \mathrm{X}_{\mathrm{CH}_{2} \mathrm{O}}$, . where two observations are pertinent. First, despite the disruption due to turbulence, the correlation remains valid for dodecane combustion (and by implication suggests that 
will also be the case for other large hydrocarbons). Second, the increased local heat release that occurs in negatively curved regions is accompanied by a proportional increase in the product of mole fractions (the correlation extends past the values obtained in a steady unstrained flat flame).

Motivated by a recent study in methane [3], which found a shift in chemical composition due to turbulence, chemical composition has been analysed through conditional means of species molar concentration conditioned on temperature. Conditional means for the primary fuel fragments are shown in Fig. 7 and other intermediate species in Fig. 8. In all cases, the black line denotes the conditional mean, and the grey regions denote one standard deviation either side of the mean. With increasing Karlovitz number, all of the primary fuel fragments generally follow the transition from the steady unstrained profile with full transport (red line) to that with unity Lewis number transport (blue line), as reported previously (see, e.g. [3]). For the intermediate species in Fig. 8, there is a departure from this behaviour. Particularly interesting is the response of molecular and atomic hydrogen; both highly mobile species. At $\mathrm{Ka}=36$, the conditional mean of molecular hydrogen has transitioned past the unity-Lewis-number profile, and the conditional mean of atomic hydrogen has moved in the opposite direction. Two species that were observed to present low-temperature activity in methane flames are $\mathrm{HO}_{2}$ and $\mathrm{H}_{2} \mathrm{O}_{2}$ [3]; it is interesting to note that $\mathrm{HO}_{2}$ drops below the unity Lewis number profile, while the $\mathrm{H}_{2} \mathrm{O}_{2}$ profile appears to be relatively insensitive to Ka.

To investigate the differences between combustion of dodecane combustion and smaller hydrocarbons in more detail, net production rates of fuel, oxidiser and key intermediates as a function of temperature are compared in Figs. 9 and 10 for methane and dodecane, respectively. For dodecane the stable intermediates discussed above are considered. Methane oxidation begins with hydrogen abstraction to produce $\mathrm{CH}_{3}$. The $\mathrm{CH}_{3}$ then reacts into three principal reaction channels. One channel represents $\mathrm{C}_{2}$ chemistry beginning with $\mathrm{C}_{2} \mathrm{H}_{6}$. A second channel is represented by $\mathrm{CH}_{2} \mathrm{O}$ (with a side branch through $\mathrm{CH}_{3} \mathrm{O}$ that then forms $\mathrm{CH}_{2} \mathrm{O}$ ). The third is a rapid transition to $\mathrm{CH}_{2}$ through $\mathrm{CH}_{2}^{*}$. For methane the fuel consumption and oxygen consumption occurs at roughly the same temperature, which is also reflected in the peak in $\mathrm{CH}_{3}$ and $\mathrm{CH}_{2}$ 
production. $\mathrm{CH}_{2} \mathrm{O}$ and $\mathrm{C}_{2} \mathrm{H}_{6}$ are formed at cooler temperatures from radicals diffusing upstream into the cooler region of the flow. For all of these species, the net production is essentially unchanged from the laminar flame solution.

The behaviour of dodecane is distinctly different. The peak in dodecane consumption occurs at around $1200 \mathrm{~K}$ whereas the peak in $\mathrm{O}_{2}$ consumption occurs near $1460 \mathrm{~K}$. Each of the key intermediates is produced where dodecane is consumed and consumed where $\mathrm{O}_{2}$ is consumed. Furthermore, unlike methane, the peak rate for each species is dramatically reduced in the turbulent case relative to the laminar flame. The response to turbulence is distinctly different than the the corresponding unity Lewis number laminar flame as indicated by the blue curve in Fig. 10. This suggests that the turbulence is beginning to disrupt the chemistry in the cool portion of the flame where the dodecane is being consumed.

\section{Conclusions}

Turbulent lean premixed dodecane/air flames are simulated in a doubly-periodic domain using detailed kinetics and transport over a range of Karlovitz numbers. Extensive thickening of thermal profiles through the flames was observed, particularly on the cool side of the flame, along with a considerable reduction in the turbulent burning speed relative to methane and hydrogen. This results from the high Lewis number of the fuel that suppresses wrinkling and leads to a negative correlation of local burning speed with curvature. Compared to methane, it was found that because the fuel decomposition pyrolysis and oxidation occurs in cooler regions of the flame, the resulting distribution of fuel fragments tends to be suseptible to mixing processes. The basic oxidation pathways of these species is largely unaffected by this process, but the magnitudes are considerably reduced. Consequently, and despite the disruption due to turbulence, the heat release in the dodecane flames was found to remain correlated with the product of $\mathrm{OH}$ and $\mathrm{CH}_{2} \mathrm{O}$ mole fractions, suggesting that this experimental diagnostic can be used with confidence in heavy hydrocarbons.

Several follow-on investigations are suggested by this study. Exploring the behaviour with a more 
detailed description of dodecane combustion would be a first step. Investigation of the dodecane combustion at higher pressures characteristic of operating conditions in realistic engines would represent a longer term goal. As part of such an investigation of high-pressure flames, it would be important to investigate the impact of nonideal behaviour as pressure increases.

\section{Acknowledgements}

JBB and MSD were supported by the DOE Applied Mathematics Research Program of the DOE Office of Advanced Scientific Computing Research under the U.S. Department of Energy Contract No. DE-AC0205CH11231. The work at USC was supported by the NSF under Grant No. CBET-1512214.

\section{References}

[1] A. J. Aspden, M. S. Day, J. B. Bell, Journal of Fluid Mechanics 680 (2011) 287-320.

[2] A. J. Aspden, M. S. Day, J. B. Bell, Proceedings of the Combustion Institute 33 (1) (2011) 1473 - 1480.

[3] A. J. Aspden, M. S. Day, J. B. Bell, Combustion and Flame 166 (2016) 266-283.

[4] H. Carlsson, R. Yu, X.-S. Bai, International Journal of Hydrogen Energy 39 (35) (2014) 20216-20232.

[5] H. Carlsson, R. Yu, X.-S. Bai, Proceedings of the Combustion Institute 35 (2) (2015) 1425-1432.

[6] M. Tanahashi, Y. Nada, N. Shiwaku, T. Miyauchi, in: Frontiers of Computational Science, 2007.

[7] Y. Shim, S. Tanaka, M. Tanahashi, T. Miyauchi, Proceedings of the Combustion Institute 33 (1) (2011) 1455-1462.

[8] B. Yenerdag, Y. Naka, M. Shimura, M. Tanahashi, in: Interntational Symposium on Turbulence and Shear Flow Phenomenon, 2015.

[9] B. Savard, B. Bobbitt, G. Blanquart, Proceedings of the Combustion Institute 35 (2) (2015) 1377-1384.

[10] B. Savard, G. Blanquart, Combustion and Flame 162 (5) (2015) 2020-2033.

[11] S. Lapointe, B. Savard, G. Blanquart, Combustion and Flame 162 (9) (2015) 3341-3355.

[12] B. Savard, G. Blanquart, Combustion and Flame 161 (6) (2014) 1547-1557.

[13] A. J. Aspden, M. S. Day, J. B. Bell, Proceedings of the Combustion Institute 35 (2) (2015) 1321 - 1329.

[14] A. J. Aspden, N. Nikiforakis, S. B. Dalziel, J. B. Bell, Comm. App. Math. Comput. Sci. 3 (1) (2008b) 101.

[15] X. You, F. N. Egolfopoulos, H. Wang, Proceedings of the Combustion Institute 32 (1) (2009) 403 - 410.

[16] A. Ern, V. Giovangigli, Combust. Sci. and Tech. 118 (1996) 387-396, see also http: //www . cmap.polytechnique. fr/www.eglib/.

[17] A. Nonaka, J. B. Bell, M. S. Day, C. Gilet, A. S. Almgren, M. L. Minion, Combust. Theory Modelling 16 (6) (2012) $1053-$ 1088.

[18] M. S. Day, J. B. Bell, Combust. Theory Modelling 4 (2000) 535-556.

[19] A. J. Aspden, accepted to the Combustion Symposium. 
[20] M. S. Day, J. B. Bell, P.-T. Bremer, V. Pascucci, V. Beckner, M. J. Lijewski, Combustion and Flame 156 (5) (2009) 1035 1045.

[21] A. J. Aspden, M. S. Day, J. B. Bell, Proceedings of the Combustion Institute 33 (1) (2011) 1463-1471.

[22] T. M. Wabel, A. W. Skiba, J. E. Temme, J. F. Driscoll, in: AIAA Aerospace Sciences Meeting, 2015. 


\begin{tabular}{l|c|c|c|c} 
Case & A & B & C & D \\
\hline$\check{u}[\mathrm{~m} / \mathrm{s}]$ & 0.226 & 0.570 & 1.18 & 2.47 \\
$\check{u} / s_{F}$ & 1.0 & 2.52 & 5.25 & 10.9 \\
$\mathrm{Ka}_{F}$ & 1.0 & 4.0 & 12 & 36 \\
$\mathrm{Da}_{F}$ & 1.0 & 0.40 & 0.19 & 0.092
\end{tabular}

Table 1: Turbulence properties for the four simulations. 


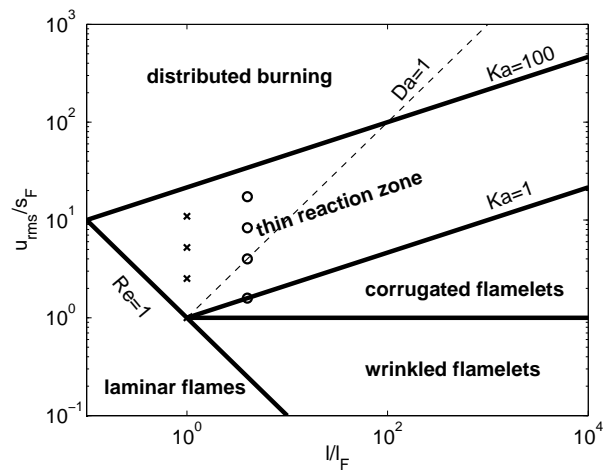

Figure 1: Regime diagram for turbulent premixed flames. The present simulations are shown by crosses, matching the Karlovitz numbers of simulations of $\mathrm{H}_{2}$ and $\mathrm{CH}_{4}$ in larger domains previously reported in $[3,13]$, which are shown by circles. 


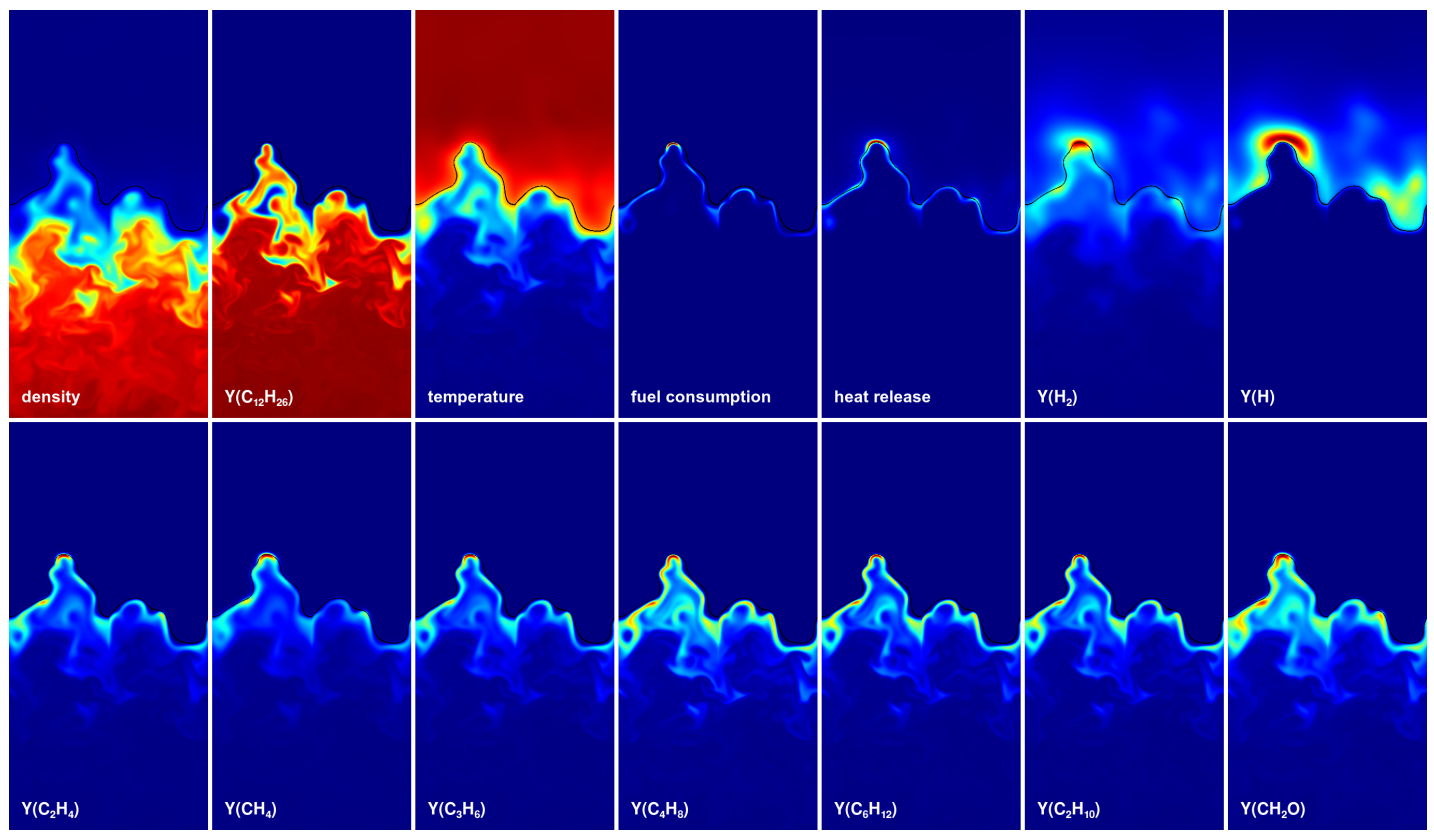

Figure 2: Two-dimensional slices through the three-dimensional data at $\mathrm{Ka}=36$. Note periodicity has been exploited to stitch together $x=0$ and $y=0$ planes to show more flame surface; each panel is two domain widths across and four high. All values have been normalised by the corresponding value in the steady unstrained flame. The black contour in each field denotes the isotherm $T=1460 \mathrm{~K}$, which corresponds the temperature of peak heat release in the steady unstrained flame. 


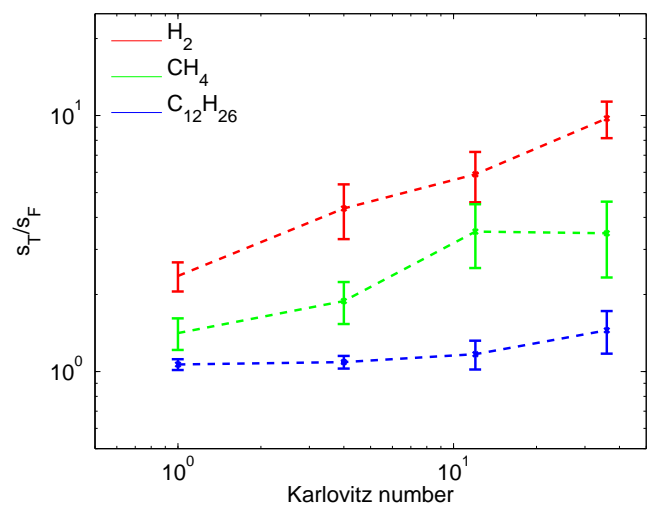

Figure 3: Consumption-based global turbulent flame speeds as a function of Karlovitz number, normalised by the steady unstrained flame speed. Crosses denote nominal averages, and the vertical lines indicate one standard deviation of the fluctuations observed once the flame has become established. 

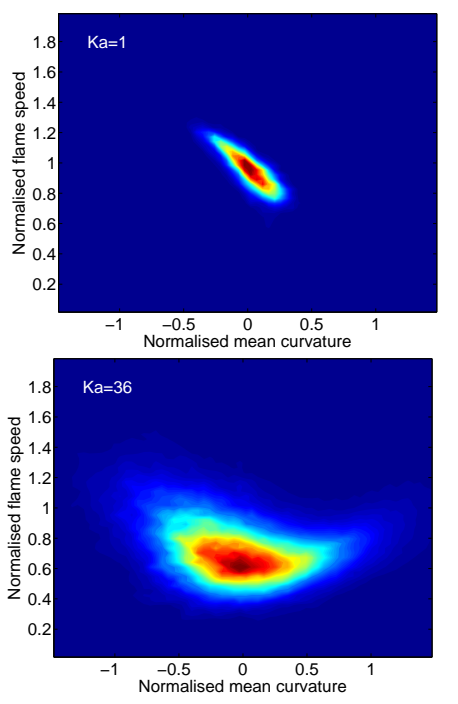

Figure 4: Joint probability density functions of normalised consumption-based turbulent flame speed $s_{T}^{c}$ and normalised mean curvature $M$ at $\mathrm{Ka}=1$ and 36 . 


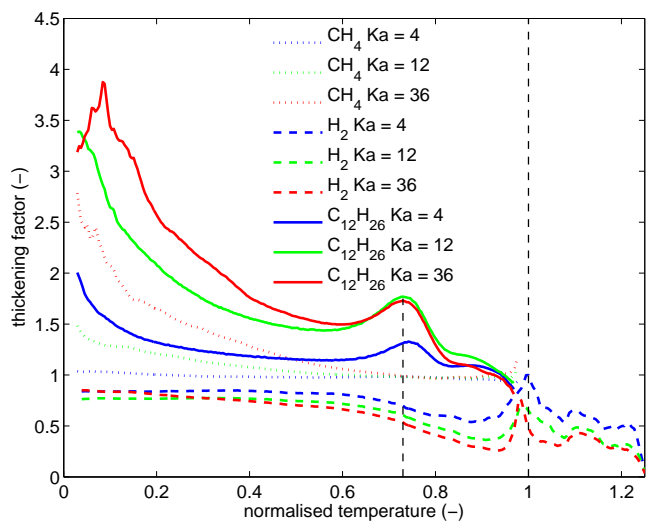

Figure 5: Thickening factor (equation 4) as a function of normalised temperature. Solid lines denote the dodecane simulations, which are compared with methane and hydrogen, represented by dotted and dashed lines, respectively. The vertical dashed line at 0.73 denotes the temperature of peak heat release in the steady unstrained flame. 

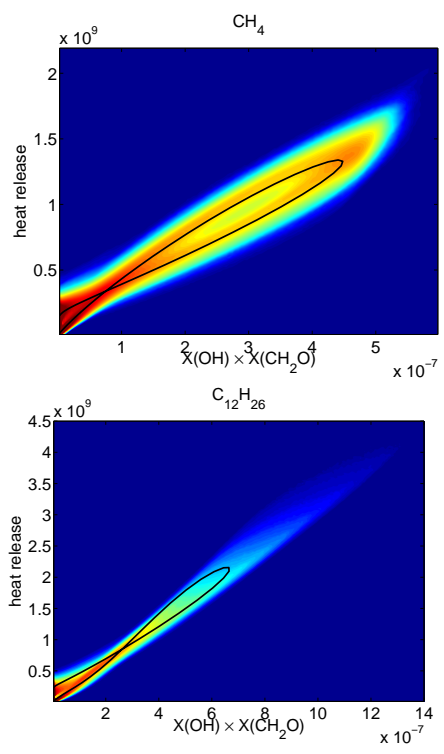

Figure 6: Joint probability density functions showing the correlation between the product of $\mathrm{OH}$ and $\mathrm{CH}_{2} \mathrm{O}$ mole fractions with heat release. The two cases are both at $\mathrm{Ka}=36$, with methane on the left and dodecane on the right. 

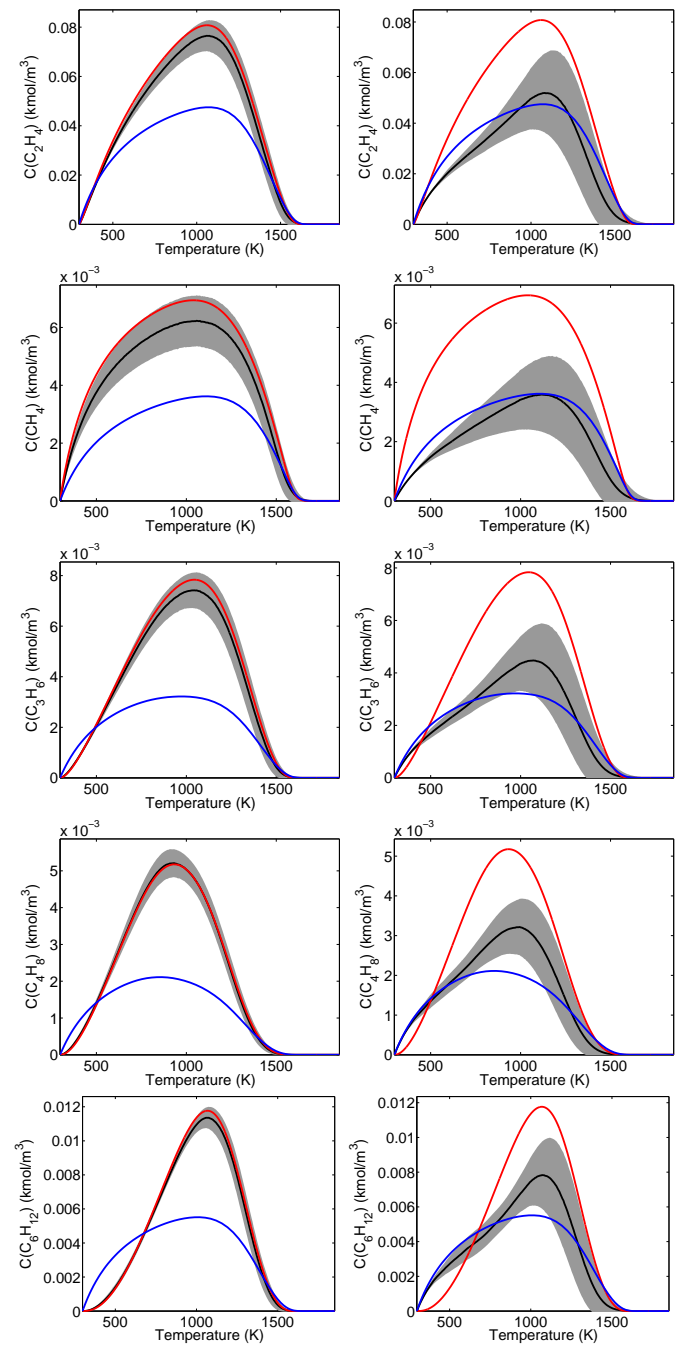

Figure 7: Conditional means of fuel fragments for $\mathrm{Ka}=1$ (left) and $\mathrm{Ka}=36$ (right). The black line denotes conditional mean, the grey area is one standard deviation either side of the mean, the red line is the steady unstrained profile, and the blue line is the unity-Lewis-number steady unstrained profile. 

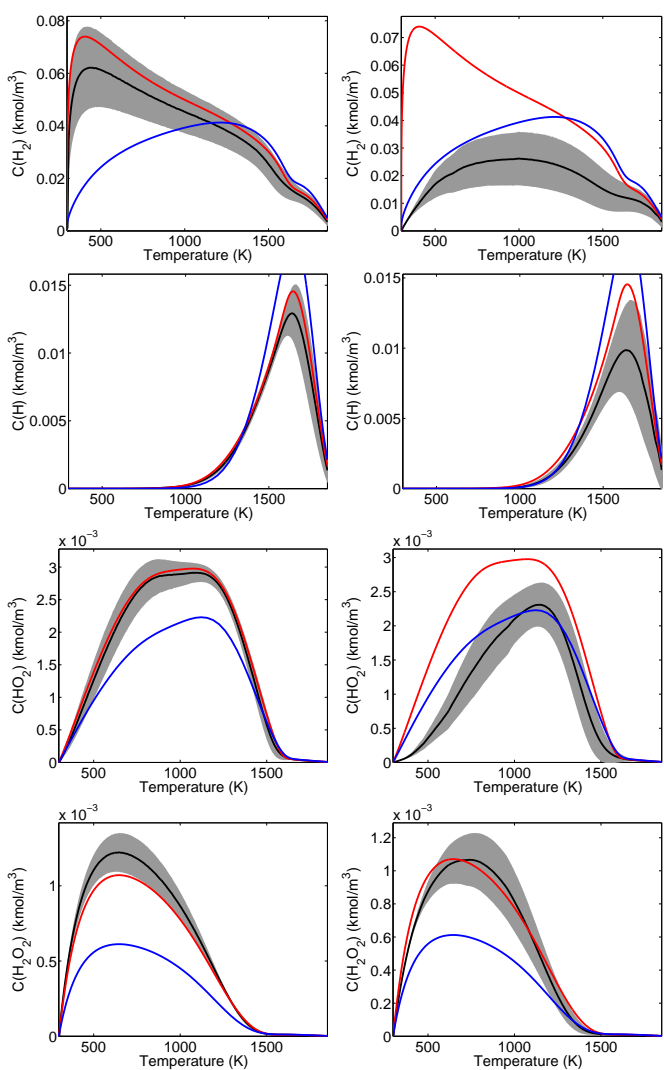

Figure 8: Conditional means of various intermediate species for $\mathrm{Ka}=1$ (left) and $\mathrm{Ka}=36$ (right); see 7 for details. 

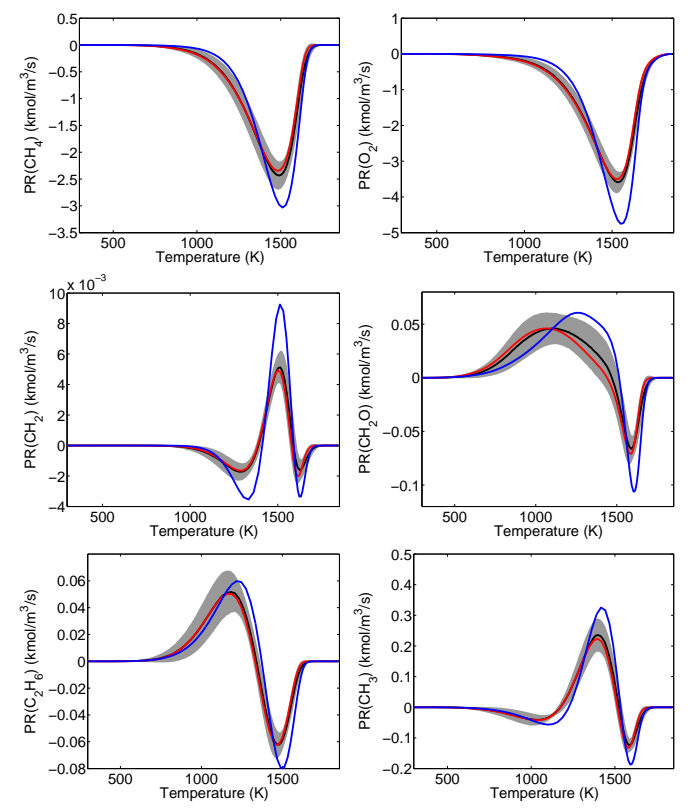

Figure 9: Conditional means of production rates $(\mathrm{Ka}=36)$ for methane as a function of temperature. 

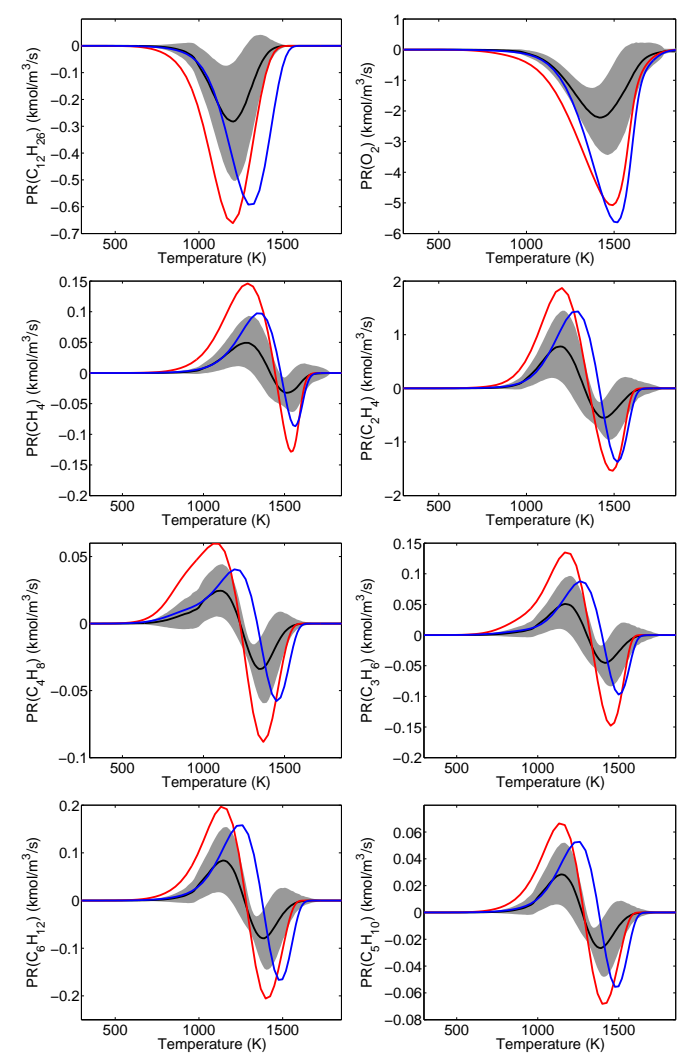

Figure 10: Conditional means of production rates $(\mathrm{Ka}=36)$ for dodecane as a function of temperature. 